\title{
Rehabilitación kinésica para pacientes con alta hospitalaria por COVID-19
}

\author{
SILVANA J. SOTO RODRÍGUEZ*
}

\section{Rehabilitation for patients after discharged from hospital by COVID-19}

By March 2020, the World Health Organization (WHO) declared the SARS-CoV-2 outbreak in a global pandemic: COVID-19. This is known as an infectious respiratory disease, which occurs mainly with symptoms and signs of dry cough, fever, tiredness and other more serious ones such as respiratory distress. This disease can cause long-term dysfunctions of different systems. Despite the fact that most of the evidence reported so far in rehabilitation and in the tools used to evaluate patients, the vast majority have been focused on the in-hospital environment, some authors, experts and international societies have shared recommendations for the work, with patients once discharged, at home, with a communitybased outlook and with remote monitoring by professionals. Based on the evidence provided and experts recommendations, a structure for evaluating the patient and an orientation in its considerations for the treatment and monitoring of patients is proposed.

Key words: COVID-19; SARS-CoV-2; Patient discharge; Hospitals; Rehabilitation; Exercise.

\section{Resumen}

El 11 de marzo de 2020, la Organización Mundial de la Salud (OMS) declaró el brote de SARSCoV-2 una pandemia mundial: COVID-19. Ésta se conoce como una enfermedad respiratoria infecciosa, que se presenta principalmente con síntomas y signos de tos seca, fiebre, cansancio y otros más graves como la dificultad respiratoria. Esta enfermedad puede causar disfunciones a largo plazo de distintos sistemas. A pesar de que la mayoría de la evidencia reportada hasta el momento en rehabilitación y en las herramientas utilizadas para evaluar a los pacientes, han sido en su gran mayoría enfocado al ambiente intrahospitalario, algunos autores, expertos y sociedades internacionales han compartido recomendaciones para el trabajo con pacientes una vez dados de alta, en sus hogares, con una mirada basada en la comunidad y con seguimientos de forma remota por los profesionales. A partir de la evidencia entregada y las recomendaciones de expertos, se propone una estructura de evaluación del paciente y una orientación en sus consideraciones para el tratamiento y seguimiento de los pacientes.

Palabras clave: COVID-19; SARS-CoV-2; Alta del paciente; Hospitales; Rehabilitación; Ejercicio.

\section{Introducción}

En diciembre de 2019, se informó de una serie de pacientes con neumonía grave de etiología desconocida en la ciudad de Wuhan, provincia de Hubei, China. Llegó a conocerse como la enfermedad del coronavirus 2019 (COVID-19), causada por el coronavirus 2 del síndrome respiratorio agudo severo (SARS-CoV-2) ${ }^{1}$. El 11 de marzo de 2020, la Organización Mundial de la Salud (OMS) declaró el brote de SARSCoV-2 una pandemia mundial. COVID-19 es una enfermedad respiratoria infecciosa, que se presenta principalmente con síntomas y signos de tos seca, fiebre, cansancio y otros síntomas más graves como la dificultad respiratoria. Esta enfermedad puede causar disfunciones a largo plazo de distintos sistemas, se han reportado

* Kinesióloga, Magíster en Fisiología Clínica del Ejercicio. Encargada Sala de Enfermedades Respiratorias del Adulto (ERA). Centro de Salud Familiar Cóndores de Chile. El Bosque. Santiago, Chile. 
alteraciones a nivel: pulmonar, cardiovascular, sistema nervioso central, hiposmia y digeusia, alteraciones de la conciencia y manifestaciones neuropsicológicas ${ }^{2,3}$.

\section{Secuelas de pacientes posterior a estadía en unidades de cuidados críticos}

Debido a la gran cantidad de sistemas invadidos por este virus, muchos pacientes han requerido soporte ventilatorio mecánico, durante varios días, por lo que es posible encontrar en ellos el síndrome post cuidados intensivos (PICS). Este síndrome se caracteriza por ${ }^{4}$ :

Deficiencias cognitivas: Memoria, atención, visuo-espacial, psicomotor, impulsividad.

Enfermedad psiquiátrica: Ansiedad, depresión, trastorno de estrés postraumático.

Impedimentos físicos: Disnea, función pulmonar deteriorada, dolor, disfunción sexual, intolerancia al ejercicio, neuropatías, debilidad muscular, paresia, fatiga severa.

Según estudios se encontraron muchos de estos síntomas y signos, incluso posterior a un año de haber presentado este síndrome ${ }^{4}$.

También los síndromes de polineuropatía por enfermedad crítica (CIP) y la miopatía por enfermedad crítica (CIM), se observaron en aproximadamente el $25-45 \%$ de los pacientes críticos, después de largas estadías en cuidados intensivos con ventilación mecánica y exhibieron complicaciones neurodegenerativas aún más graves, incluyendo parálisis flácida y simétrica, debilidad muscular de las extremidades y las vías respiratorias, síndrome de respuesta inflamatoria sistémica con falla multiorgánica ${ }^{5,6,7}$.

\section{Desafíos posibles de enfrentar en un paciente con una infección por COVID-19}

Se ha reportado a nivel mundial, información respecto al daño sistémico producido por el COVID-19 en los pacientes ${ }^{2,5,8,9}$ :

Función respiratoria: El daño pulmonar conduce al deterioro del intercambio gaseoso alvéolo - capilar y a la disminución de la función de ventilación pulmonar. Como resultado, muchos pacientes informaron disnea y opresión torácica. La fibrosis pulmonar es otro factor importante que podría afectar la función pulmonar a largo plazo.

Función cardíaca: Los niveles de troponina cardíaca sérica I (cTnI), creatina quinasa $(\mathrm{CK})$, isoenzima de creatina quinasa (CKMB), lactato deshidrogenasa (LDH) y $\alpha$-hidroxibutirato deshidrogenasa ( $\alpha \mathrm{HBDH})$ aumentaron significativamente en algunos pacientes graves y críticos. Los marcadores séricos elevados, a menudo iban acompañados de taquicardia, que no era proporcional al aumento de la temperatura corporal. $\mathrm{Si}$ estos daños miocárdicos provocaran una disfunción a largo plazo merece atención.

Funciones de otros sistemas: Los estudios sugirieron que los sistemas hepático, renal, cerebral, nervioso e inmune de muchos pacientes críticos tienen agregación de virus y daño tisular, así como la disfunción correspondiente. El resultado de esta disfunción orgánica aún no se ha investigado.

Función motora: La debilidad y la disminución de la capacidad de ejercicio son los síntomas y la disfunción más comunes. Las causas de la debilidad pueden atribuirse a la disminución de la resistencia al ejercicio asociada con la disfunción cardiopulmonar, y a la atrofia muscular causada por la inmovilización a largo plazo en pacientes graves, e incluso puede estar relacionada con la invasión muscular por el virus.

\section{Rehabilitación de pacientes post hospitalización}

Los sistemas de salud a nivel global han debido reaccionar de manera rápida, adaptándose a las demandas de la población y aunque gran parte de la pandemia aún está evolucionando, ya ha surgido preocupación acerca de las necesidades de rehabilitación posteriores a COVID-19.

Algunos trabajos presentaron sus estrategias para la rehabilitación pulmonar durante la

fase aguda intrahospitalaria, basándose en experiencia y evidencia previa en rehabilitación, debido al desconocimiento actual de las necesidades específicas de estos pacientes al alta, a mediano y/o largo plazo ${ }^{10-13}$.

Respecto a las herramientas de evaluación, si bien no hay estándares descritos, distintos autores reportaron sus elecciones al evaluar a estos pacientes ${ }^{14-17}$. A continuación, se presenta lo comunicado (Tabla 1).

Debido a la posibilidad de encontrar múltiples disfunciones (alteraciones de piso pélvico, traumatológicas, neurológicas, fonoaudiológicas, psicológicas, etc.), se debe contar con un equipo multidisciplinario que pueda evaluar además de forma específica estas alteraciones.

En relación con la prescripción de la rehabilitación, la mayoría de la evidencia se centra en la rehabilitación "intrahospitalaria" previo al alta. 
Tabla 1. Herramientas para evaluación de la rehabilitación post-COVID-19

\begin{tabular}{|c|c|}
\hline Área a evaluar & Herramientas de evaluación \\
\hline Calidad de vida & $\begin{array}{ll}\text { - } & \mathrm{SF}-36 \\
\text { - } & \text { COPD Assessment test (CAT) } \\
\text { - } & \text { EuroQol-5D (EQ-5D) }\end{array}$ \\
\hline Disnea & $\begin{array}{l}\text { - Modified Medical Research Council (mMRC) } \\
\text { - Borg modificado } \\
\text { - Borg original } \\
\text { - Test del habla }\end{array}$ \\
\hline Evaluación funcional & $\begin{array}{l}\text { - Índice de Barthel } \\
\text { - Medición de la independencia funcional (FIM) } \\
\text { - Short Physical Performance Battery (SPPB; incluye velocidad de marcha, equilibrio y } \\
\text { potencia de extremidad inferior) } \\
\text { - Post-COVID-19 Functional Status Scale (PCFS) }\end{array}$ \\
\hline Evaluación aeróbica & $\begin{array}{l}\text { - Test de marcha de } 6 \mathrm{~min} \\
\text { - Sit to stand test }(1 \mathrm{~min})\end{array}$ \\
\hline Fuerza & $\begin{array}{l}\text { - Medical Research Council (MRC), utilizado intrahospitalario } \\
\text { - Escala de Daniels } \\
\text { - Dinamometría }\end{array}$ \\
\hline Equilibrio & $\begin{array}{l}\text { - Test Time up and go } \\
\text { - Test de estación unipodal } \\
\text { - Test de Berg }\end{array}$ \\
\hline Dolor & - Escala visual análoga (EVA) \\
\hline Cognitivo & - Minimental abreviado o extendido (MMSE) \\
\hline
\end{tabular}

Sin embargo, expertos y sociedades internacionales han dado recomendaciones, basados en la comunidad y realizados de forma remota ${ }^{18-23}$. A continuación, lo recopilado se resume en la Tabla 2.

\section{Consideración clínica}

De la mano de la rehabilitación remota, debe acompañar la constante educación a la familia y al paciente, sobre la auto-regulación en los ejercicios, de identificar signos de detención, ya sea mediante el uso de la escala de Borg, el test del habla, o bien el uso de dispositivos más objetivos como un oxímetro de pulso que permita mantener a la persona dentro de rangos "objetivos de seguridad".

Si bien un criterio de detención o contraindicación del ejercicio es la frecuencia cardíaca de 100 latidos por minuto o más, es muy probable que debido al nivel de desacondicionamiento aeróbico, muchos de estos pacientes se encuentren en esta condición inicial, algo que distintos expertos han confirmado en sus experiencias a través de variadas instancias de videoconferencias, por lo cual, este criterio está sujeto a discusión constante y debería evaluarse con esa mirada por los terapeutas en sus programas de entrenamiento, siempre considerando rangos de protección, y sin que esto implique una limitación al trabajo con el paciente.

Entendiendo la capacidad de comprensión variada de los pacientes y la dificultad de prescripción y monitorización de estos por parte de los terapeutas, las pautas de ejercicio (considerando series, repeticiones, cargas, frecuencia, etc.) deberían entregarse cuando el paciente pueda comprender y aplicar adecuadamente la indicación. Si el paciente o la familia no tienen esta capacidad de supervisión y aplicación de la pauta, podría el programa basarse en el uso de ejemplos cotidianos y realizables fácilmente, considerando las tablas del gasto energético en "MET". Los MET son la razón entre el metabolismo de una persona durante la realización de un trabajo y su metabolismo basal. Un MET se define como el costo energético de estar sentado 
Tabla 2. Características y descripción de las prescripciones de ejercicio usadas en rehabilitación post-COVID-19

\begin{tabular}{|c|c|}
\hline Características & Descripción \\
\hline Frecuencia & $\begin{array}{l}\text { - Aeróbico: } \\
\text { 3-5 veces por semana } \\
\text { 5-7 veces por semana } \\
\text { - Fuerza: } \\
\text { 2-3 veces por semana }\end{array}$ \\
\hline Intensidad & $\begin{array}{l}\text { - Aeróbico: } \\
\text { Menor al } 60 \% \text { de frecuencia cardíaca máxima } \\
\text { Entre el } 50-70 \% \text { de frecuencia cardíaca de reserva } \\
\text { 3-4 Borg disnea } \\
\text { Intensidades leves a moderadas } \\
\text { - Fuerza: } \\
70-80 \% \text { de } 1 \text { repetición máxima } \\
\text { 8-12 repeticiones máximas, } 1-3 \text { grupos musculares } \\
\text { Uso de bandas elásticas } \\
\text { Progreso de un } 5-10 \% \text { de carga semanalmente }\end{array}$ \\
\hline Tiempo & $\begin{array}{l}\text { - 6-8 semanas y más } \\
\text { - 20-30 min cada sesión } \\
\text { - } 10-15 \text { min al menos por sesión } \\
\text { - Posible necesidad de ejercitarse más de una vez al día }\end{array}$ \\
\hline Tipo & $\begin{array}{l}\text { Respiratorio: } \\
\text { Ejercicios respiratorios con válvula de resistencia (inspiratory muscle training IMT, Power } \\
\text { Breath electrónico) } \\
\text { Ejercicios de tos } \\
\text { Ejercicios diafragmáticos con pesos en pared abdominal en posición supina (1-3 kg) } \\
\text { Técnica del ciclo activo de respiración (ACBT) } \\
\text { Respiración de labios fruncidos } \\
\text { - Aeróbico: } \\
\text { Continuo } \\
\text { Interválico } \\
\text { Intermitente } \\
\text { Caminata lenta o rápida, nadar, trotar } \\
\text { - Fuerza: } \\
\text { Resistencia } \\
\text { Equilibrio, core, otros }\end{array}$ \\
\hline $\begin{array}{l}\text { Contraindicaciones } \\
\text { o detención de } \\
\text { tratamiento }\end{array}$ & $\begin{array}{l}\text { - Frecuencia cardíaca }>100 \text { latidos/min (relativo) } \\
\text { - Presión arterial }<90 / 60 \mathrm{mmHg},>140 / 90 \mathrm{mmHg} \text { o fluctuaciones de la presión arterial que } \\
\text { exceden los } 20 \mathrm{mmHg} \text { basales } \\
\text { - Saturación de oxígeno de la hemoglobina } \leq 95 \% \text { en pacientes sin enfermedad respiratoria } \\
\text { crónica de base } \\
\text { - Saturación de } \mathrm{O}_{2}<88 \% \text { o por debajo de saturación basal de paciente con enfermedad res- } \\
\text { piratoria crónica } \\
\text { - Mareos, dolor de cabeza y otros síntomas incómodos (la fatiga no se alivia después del } \\
\text { descanso, opresión o dolor en el pecho, dificultad para respirar, tos severa, visión borrosa, } \\
\text { palpitaciones, sudoración, inestabilidad, etc.) } \\
\text { - Frente a un comportamiento con parámetros como los descritos, debe suspender inmedia- } \\
\text { tamente, reevaluar y ajustar el plan de tratamiento } \\
\text { - No realizar ejercicios de intensidad vigorosa }\end{array}$ \\
\hline
\end{tabular}


tranquilamente y es equivalente a un consumo de $1 \mathrm{kcal} / \mathrm{kg} / \mathrm{h}$. En estos pacientes, dependiendo de su situación a la evaluación, se recomiendan actividades que presenten hasta 5,9 MET, que se considera una actividad de moderado esfuerzo. Para esto podrían realizarse prescripciones basa- das en indicaciones de la $\mathrm{OMS}^{24}$ (Tabla 3).

Siendo más específicos y explicativos con las prescripciones para los pacientes, puede recurrirse a tablas utilizadas por grupos de expertos como la American College Sport Medicine $(\mathrm{ACSM})^{25}$ (Tabla 4).

Tabla 3. Equivalencias en MET de actividades diarias, según la OMS

\begin{tabular}{|lll|}
\hline MET & Actividades a las que equivale & Explicación \\
Aproximado & Caminar a paso rápido & "Requiere un esfuerzo \\
de 3-6 & Bailar & moderado, que acelera de \\
& Jardinería & forma perceptible el ritmo \\
& cardiaco y la respiración" \\
& Ir de compras & \\
& Participación en juegos y deportes con niños y paseos con animales & \\
& domésticos & \\
& Trabajos de construcción generales (tejados, pintar, etc. $)$ & \\
& Desplazamiento de cargas moderadas $(<20 \mathrm{~kg})$ & \\
\hline
\end{tabular}

Tabla 4. Equivalencia en MET de actividades diarias

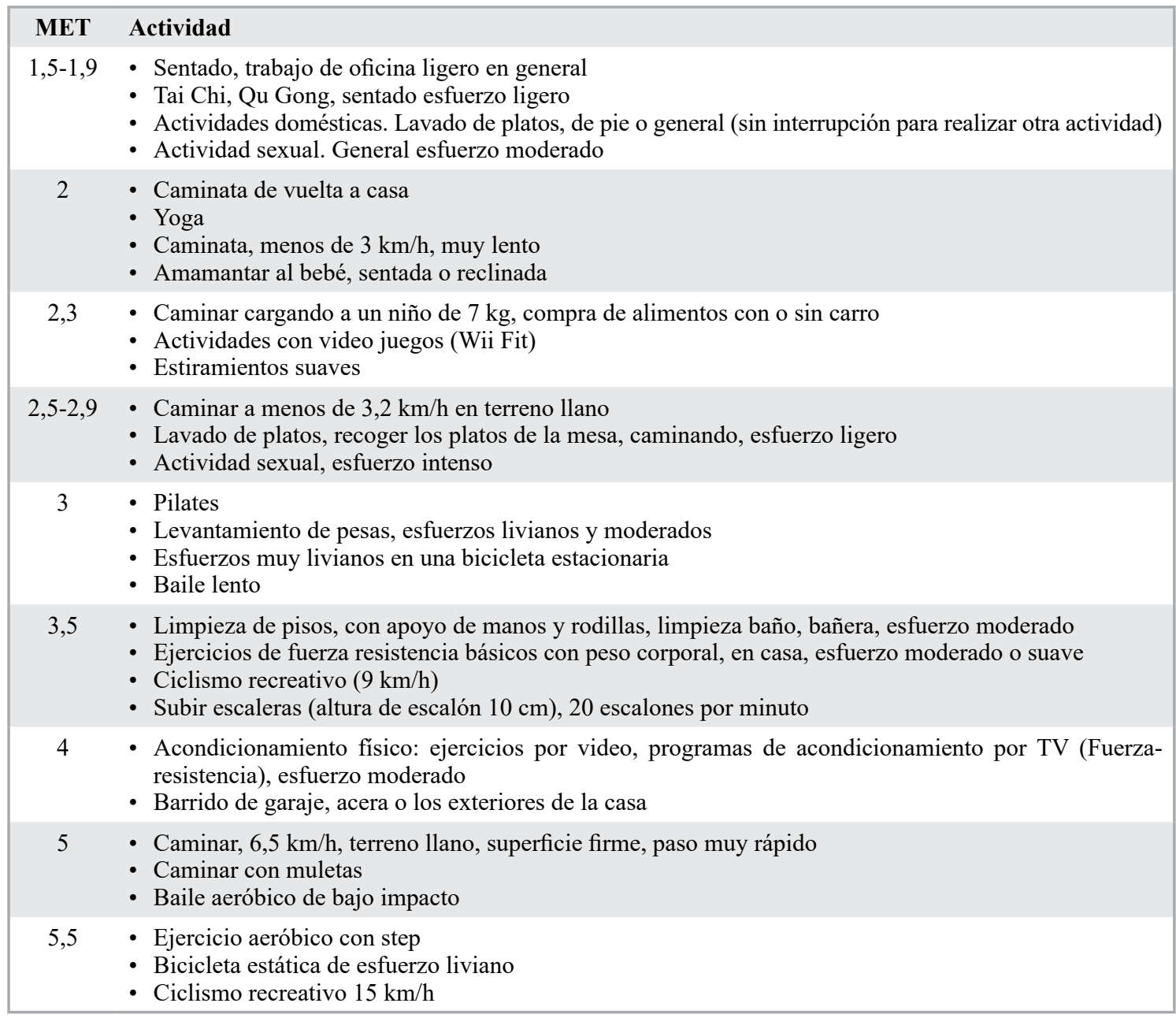




\section{Desafíos por enfrentar a nivel país}

Las demandas actuales del sistema sanitario están forzando a los distintos sectores de salud a actuar con sobre esfuerzos en relación con la cantidad de funciones que están realizando y el personal que tienen a disposición. A partir de esta situación, es fundamental el rol de los equipos multidisciplinarios de rehabilitación en la atención primaria, quienes recibirán a los pacientes dados de alta de los hospitales, y es de vital importancia que este equipo participe activamente en la planificación de estos programas, a nivel estratégico y operacional, siendo flexibles al contexto cambiante de la pandemia actual y actualizándose permanentemente con las nuevas posibilidades terapéuticas que surjan a diario, adaptándose a las necesidades específicas de sus pacientes.

\section{Propuesta}

A modo de lograr una clasificación del deterioro funcional de los pacientes y a partir de eso, orientarse inicialmente en la rehabilitación, se propone considerar la condición funcional del paciente (medido a través de alguna escala específica como Barthel) y/o el uso tablas de actividades cotidianas, medidas en MET (Figuras 1 y 2).

\section{Factores protectores}

La educación a la familia y al paciente durante la rehabilitación y al alta debe enfocarse en los efectos protectores que pueden lograrse a través de los estilos de vida saludable y que son importantes en este contexto COVID-19, manteniendo actividad física regular ${ }^{22,27}$, alcohol y $\operatorname{drogas}^{27}$, llevando una alimentación saludable ${ }^{28}$, utilizando técnicas de reducción de estrés ${ }^{29}$, evitando el consumo del tabaco ${ }^{27,30}$ y reduciendo el comportamiento sedentario ${ }^{31,32,33,34}$.

A pesar de que se desconoce el efecto específico de la actividad física en el COVID-19, existen recomendaciones por parte de los expertos, basándose en el conocimiento de la modulación en el sistema inmune y en la contribución en el balance pro y antiinflamatorio. Se ha demostrado que tener una alta capacidad cardio-respiratoria, ajustada por sexo y edad, y mantener actividad física regular ${ }^{35}$ (según recomendaciones de guías como la American College Sport Medicine), mejoran la respuesta inmune a la vacunación, disminuyen la inflamación crónica de bajo grado $\mathrm{y}$ mejoran varios indicadores inmunes en varias enfermedades (cáncer, VIH, enfermedad cardiovascular, diabetes, limitaciones cognitivas y obesidad) $)^{36,37}$.

Por lo anteriormente descrito, es necesario implementar programas que consideren las variables anteriores, sobre todo en el traspaso de

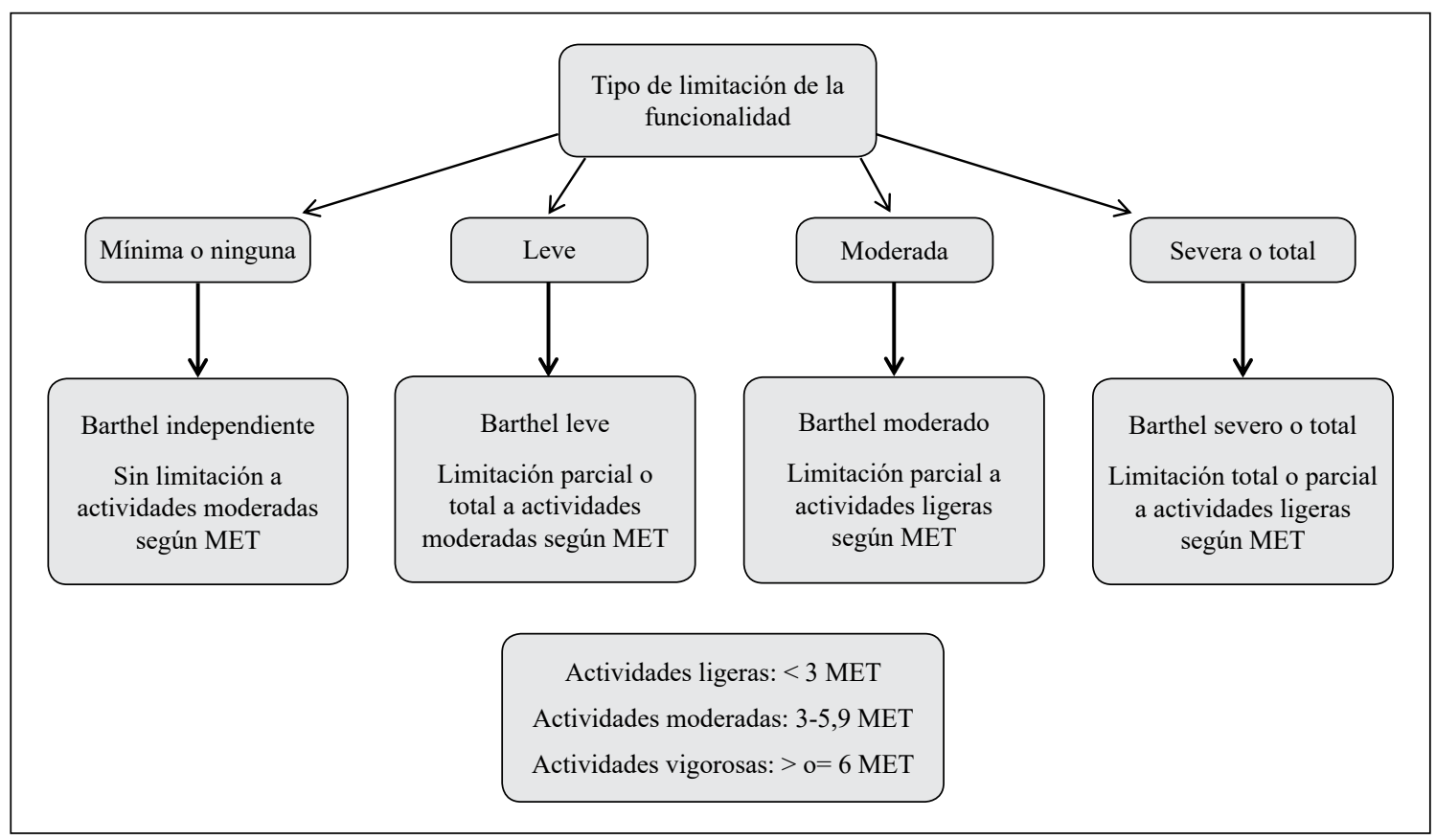

Figura 1. Tipos de limitación de la funcionalidad según escala de Barthel. 


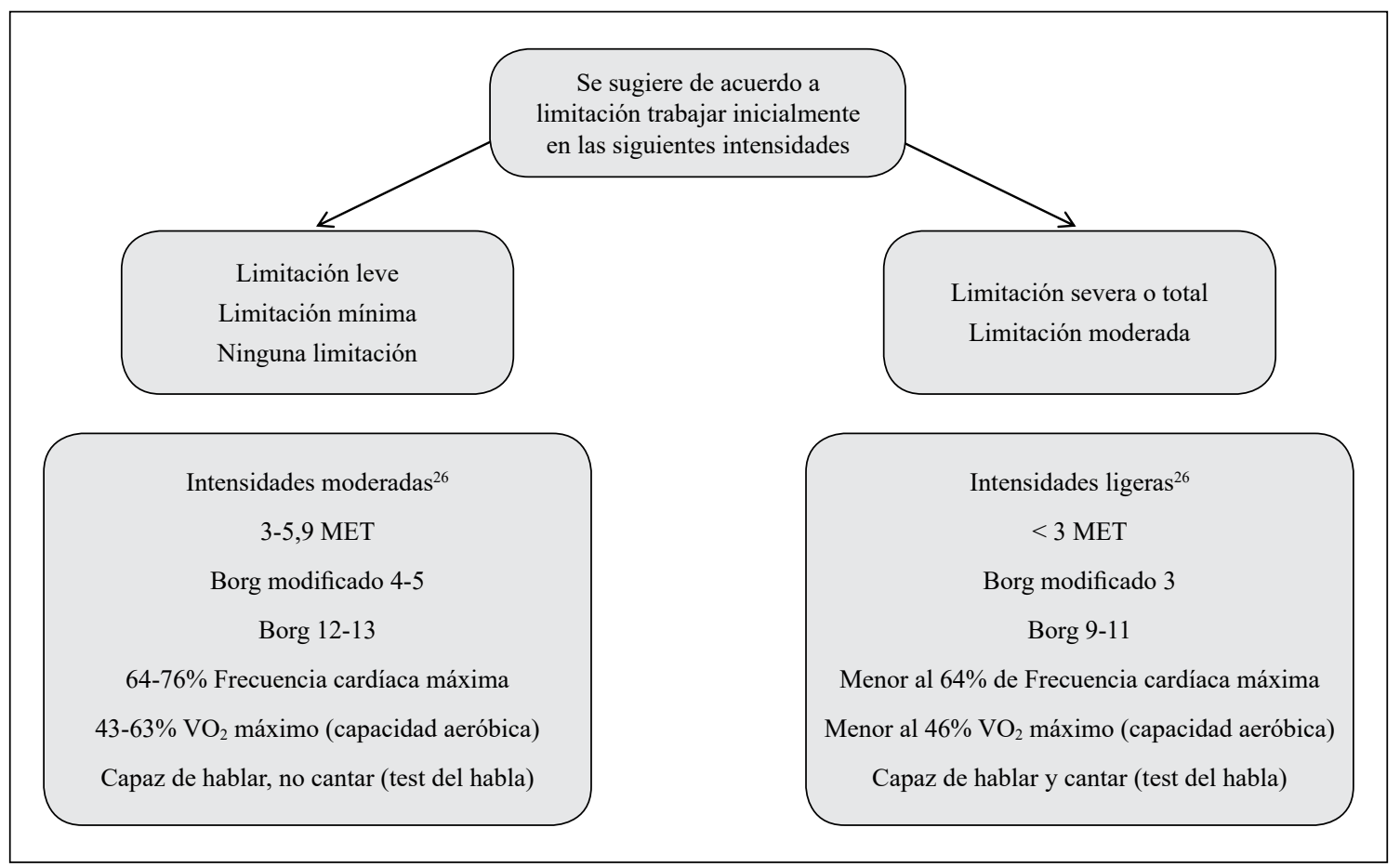

Figura 2. Intensidad del ejercicio sugerida según limitación funcional.

información al paciente y su familia, para así, no solo lograr recuperar sus funciones diarias, sino también prevenir o reducir las posibles complicaciones futuras, por el largo período de inactividad al que estuvieron sometidos.

\section{Agradecimientos}

La autora expresa agradecimientos a su equipo de trabajo y en especial a su dirección y subdirección, Marlon Leal Sepúlveda y Paula Leclerc Vargas, quienes la motivaron en esta iniciativa y además confiaron en los esfuerzos técnicos y humanos que permitieron su desarrollo.

\section{Bibliografía}

1.- LAUER SA, GRANTZ KH, BI Q, JONES FK, ZHENG Q, MEREDITH HR, et al. The Incubation Period of Coronavirus Disease 2019 (COVID-19) From Publicly Reported Confirmed Cases: Estimation and Application. Ann Intern Med. 2020; 172 (9): 577-82. doi: 10.7326/M20-0504.

2.- BRUGLIERA L, SPINA A, CASTELLAZZI P, CIMINO P, TETTAMANTI A, HOUDAYER E, et al. Rehabilitation of COVID-19 patients. J Rehabil Med.
2020; 15; 52 (4): jrm00046. doi: 10.2340/165019772678.

3.- ZHOU M, ZHANG X, QU J. Coronavirus disease 2019 (COVID 19): a clinical update. Front Med. 2020; 14 (2): 126-35. doi: 10.1007/s11684-020-0767-8.

4.- JAFFRI A, JAFFRI UA. Post-Intensive care syndrome and COVID-19: crisis after a crisis?. Heart Lung. 2020; 49 (6): 883-4. doi:10.1016/j.hrtlng.2020.06.006.

5.- STAM H, STUCKI G, BIRKENBACHER J. Covid-19 and Post Intensive Care Syndrome: A Call for Action. J Rehabil Med. 2020; 15; 52 (4): jrm00044. doi: 10.2340/16501977-2677.

6.- DESAI SV, LAW TJ, NEEDHAM DM. Long-term complications of critical care. Crit Care Med. 2011; 39: 371-9. doi: 10.1097/CCM.0b013e3181fd66e5.

7.- ZHOU C, WU L, NI F, JI W, ZHANG H. Critical illness polyneuropathy and myopathy: a systematic review. Neural Regen Res. 2014; 9: 101-10. doi: 10.4103/1673-5374.125337.

8.- LI J. Rehabilitation management of patients with Covid-19. Lessons learned from the first experiences in China. Eur J Phy Rehabil Med. 2020; 56 (3):335-38. doi: 10.23736/S1973-9087.20.06292-9.

9.- HELMS J, KREMER S, MERDJI H, CLERE-JEHL $\mathrm{R}$, SCHENCK M, KUMMERLEN C, et al. Neurologic Features in Severe SARS-CoV-2 Infection. N Engl J Med. 2020; 4; 382 (23): 2268-70. doi: 10.1056/ NEJMc2008597. 
10.- CHINESE ASSOCIATION OF REHABILITATION MEDICINE; RESPIRATORY REHABILITATION COMMITTEE OF CHINESE ASSOCIATION OF REHABILITATION MEDICINE; CARDIOPULMONARY REHABILITATION GROUP OF CHINESE SOCIETY OF PHYSICAL MEDICINE AND REHABILITATION. Recommendations for respiratory rehabilitation of COVID-19 in adult. Chinese Journal of Tuberculosis and Respiratory disease 2020; 12; 43(4): 308-14. Chinese. doi: 10.3760/ cma.j.cn112147-20200228-00206.

11.- LAZZERI M, LANZA A, BELLINI R, BELLOFIORE A, CECCHETTO S, COLOMBO A, et al. Respiratory physiotherapy in patients with COVID-19 infection in acute setting: a Position paper of the Italian Association of Respiratory Physiotherapists (ARIR). Monaldi Arch Chest Dis. 2020; 90 (1). doi: 10.4081/ monaldi.2020.1285.

12.- MODRYKAMIEN AM. The ICU follow-up clinic: a new paradigm for intensivists. Respir Care. 2012; 57:764-72. doi: 10.4187/respcare.01461.

13.- SEVIN CM, JACKSON JC. Post-ICU clinics should be staffed by ICU clinicians. Crit Care Med. 2019; 47: 268-72. doi: 10.1097/CCM.0000000000003535.

14.- LIU K, ZHANG W, YANG Y, ZHANG J, LIA Y, CHEND Y. Respiratory rehabilitation in elderly patients with COVID-19: A randomized controlled study. Complement Ther Clin Pract. 2020; 39: 101166. doi: 10.1016/j.ctcp.2020.101166.

15.- SMITH SR, JENQ G, CLAFLIN T, MAGNANT C, HAIG AJ, HURVITZ E. Proposed Workflow for Rehabilitation in a Field Hospital Setting During the COVID-19 Pandemic. PM R. 2020; 12 (8): 823-28. doi: 10.1002/pmrj.12405.

16.- PAOLINELLI C, GONZÁLEZ P, DONIEZ ME, DONOSO T, SALINAS V. Clinical use and inter rater agreement in the application of the functional independence measure. Rev Med Chile 2001; 129 (1): 23-31. doi:10.4067/S0034-98872001000100004.

17.- KLOK FA, BOON GJAM, S BARCO, ENDRES M, GEELHOED JJM, KNAUSS S, et al. The Post-COVID-19 Functional Status (PCFS) Scale: a tool to measure functional status over time after COVID-19. Eur Respir J. 2020; 2; 56 (1): 2001494. doi: 10.1183/13993003.01494-2020.

18.- LI J. Effect and enlightenment of rehabilitation medicine in COVID-19 management. European Journal of Physical and Rehabilitation Medicine 2020. doi: 10.23736/S1973-9087.20.06292-9.

19.- BABU AS, ARENA R, OZEMEK C, LAVIE CJ. COVID-19: A Time for Alternate Models in Cardiac Rehabilitation to Take Centre Stage. Can J Cardiol. 2020; 36 (6): 792-94. doi: 10.1016/j.cjca.2020.04.023.

20.- NEGRINI S, KIEKENS C, BERNETTI A, CAPECCI M, CERAVOLO MG, LAVEZZI S, et al. Telemedicine From Research to Practice During the
Pandemic. "Instant Paper From the Field" on Rehabilitation Answers to the Covid-19 Emergency. Eur J Phys Rehabil Med. 2020; 56 (3): 327-30. doi: 10.23736/ S1973-9087.20.06331-5.

21.- NIEMAN DC, WENTZ LM. The compelling link between physical activity and the body's defense system. J Sport Health Sci. 2019; 8 (3): 201-17. doi: 10.1016/j. jshs.2018.09.009

22.- MOHAMED A, ALAWNA M. Role of increasing the aerobic capacity on improving the function of immune and respiratory systems in patients with coronavirus (COVID-19): A review. Diabetes Metab Syndr. 2020; 14 (4): 489-96. doi: 10.1016/j.dsx.2020.04.038.

23.- YANGA L, YANG T. Pulmonary Rehabilitation for Patients with Coronavirus Disease 2019 (COVID-19). Chronic Dis Transl Med. 2020; 14; 6 (2): 79-86. doi: 10.1016/j.cdtm.2020.05.002.

24.- Who.int. World Health Organization. 2020. Physical activity intensity. Disponible en: https:/www.who.int/ dietphysicalactivity/physical_activity_intensity/es/ . [Consultado 06 de junio 2020].

25.- ABELLÁN J, SAINZ DE BARANDA ANDUJAR P, ORTÍN E. Ejercicio para la mejora de la resistencia cardiorrespiratoria. En: Abellán J, Sainz de Baranda Andujar P, Ortín E. Guía para la Prescripción de Ejercicio Físico en Pacientes con Riesgo Cardiovascular. $2^{\text {a }}$ Edición. SEH - LELHA. Murcia, España. 2011; 73-5.

26.- ACSM: POSITION STAND. Quantity and Quality of Exercise for Developing and Maintaining Cardiorespiratory, Musculoskeletal, and Neuromotor Fitness in Apparently Healthy Adults: Guidance for Prescribing Exercise. 2011. Med Sci Sports Exerc. 2011; 43 (7): 1334-59. doi: 10.1249/MSS.0b013e318213fefb.

27.- SILVER J. Prehabilitation could save lives in a pandemic. BMJ. 2020; 369: m1386. doi: 10.1136/bmj.m1386

28.- SCHWINGSHACKL L, HOFFMANN G. Mediterranean dietary pattern, inflammation and endothelial function: a systematic review and meta-analysis of intervention trials. Nutr Metab Cardiovasc Dis. 2014; 24: 929-39. doi: 10.1016/j.numecd.2014.03.003

29.- GOYAL M, SINGH S, SIBINGA EMS, GOULD NF, ROWLAND-SEYMOUR A, SHARMA R, et al. Meditation programs for psychological stress and wellbeing: a systematic review and meta-analysis. JAMA Intern Med. 2014; 174: 357-68. doi:10.1001/jamainternmed.2013.13018

30.- LEMMENS V, OENEMA A, KNUT IK, BRUG J. Effectiveness of smoking cessation interventions among adults: a systematic review of reviews. Eur J Cancer Prev. 2008; 17: 535-44. doi: 10.1097/ CEJ.0b013e3282f75e 48 .

31.- BISWAS A, OH PI, FAULKNER GE, BAJAJ RR, SILVER MA, MITCHELL MS, et al. Sedentary time and its association with risk for disease incidence, mortality, and hospitalization in adults: a systematic review 
and meta-analysis. Ann Intern Med. 2015; 162: 123-32. doi: 10.7326/M14-1651.

32.- WILMOT EG, EDWARDSON CL, ACHANA FA., DAVIES MJ, GORELY T, GRAY LJ, et al. Sedentary time in adults and the association with diabetes, cardiovascular disease and death: systematic review and meta-analysis. Diabetologia. 2012; 55 (11): 2895-905. doi: 10.1007/s00125-012-2677-z.

33.- DUNSTAN DW, KINGWELL BA, LARSEN R, HEALY GN, CERIN E, HAMILTON MT, et al. Breaking up prolonged sitting reduces postprandial glucose and insulin responses. Diabetes Care. 2012; 35 (5): 976-83. doi: 10.2337/dc11-1931.

34.- HOWARD BJ, FRASER SF, SETHI P, CERIN E, HAMILTON MT, OWEN N, et al. Impact on hemostatic parameters of interrupting sitting with intermittent activity. Med Sci Sports Exerc. 2013; 45 (7): 1285-91. doi: 10.1249/MSS.0b013e318285f57e.

35.- AMERICAN COLLEGE OF SPORT MEDICINE. 2020. Ejercicio, inmunidad y la pandemia del COVID-19. Disponible en: https:/www.acsm.org/ blog-detail/acsm-blog/2020/04/16/ejercicio-inmunidady-la-pandemia-del-covid-19 [Consultado el 6 de agosto 2020].

36.- KLARLUND PB, STEENSBERG A, SCHJERLING

P. Muscle-derived interleukin-6: possible biological effects. J Physiol. 2001; 536 (Pt 2): 329-37. doi: 10.1111/j.1469-7793.2001.0329c. xd.

37.- DA SILVEIRA MP, DA SILVA FAGUNDES KK, BIZUTI MR, MATHEUS RIBEIRO SE, ROSSI RC, DE RESENDE E SILVA DT. Physical exercise as a tool to help the immune system against COVID-19: an integrative review of the current literature. Clin Exp Med. 2020; 29: 1-14. doi: 10.1007/s10238-020-00650-3.

Correspondencia a:

Klga. Silvana J. Soto Rodríguez

Centro de Salud Familiar Cóndores de Chile.

Augusto D'Halmar 555, El Bosque. Santiago, Chile.

Email: silvana.soto.r@gmail.com 